\title{
Reicht Lithium im Trinkwasser zur Demenzprophylaxe?
}

\begin{abstract}
Dänische Wissenschaftler finden einen Zusammenhang zwischen Lithium, das mit dem Trinkwasser aufgenommen wird, und der Demenzinzidenz. Allerdings ist die Assoziation alles andere als linear. Basis ihrer bevölkerungsgestützten Studie sind die über einen Zeitraum von 28 Jahren gesammelten Daten von mehr als 800.000 Dänen.
\end{abstract}

\) Bereits 2009 gab es Hinweise, dass durch Leitungswasser aufgenommenes Lithium suizidpräventiv wirkt. Jetzt haben Wissenschaftler um Dr. Lars Vedel Kessing vom Reichshospital in Kopenhagen erstmals den Zusammenhang mit der Demenzinzidenz in Dänemark überprüft. Für diese Studie wurden die Daten von fast 74.000 Patienten mit einer Demenz sowie von fast 734.000 Personen ohne Demenz als Kontrollgruppe ausgewertet. Das mediane Alter der Studienteilnehmer lag bei 80 Jahren. Von 2009 bis 2010 sowie im Jahr 2013 wurden in 151 Wasserwerken des Landes Proben gezogen, um den durchschnittlichen Lithiumgehalt zu bestimmen. Der Durchschnittswert lag bei 11,6 $\mu \mathrm{g} / 1$, mit einer Spannweite von 0,6 $\mu \mathrm{g} / \mathrm{l}$ im Westen Dänemarks bis zu mehr als knapp $31 \mathrm{\mu g} / \mathrm{l}$ im Osten des Landes.

Aus den Auswertungen geht hervor, dass Menschen, bei denen eine Demenz diagnostiziert worden war, durch das Trinkwasser einer durchschnittlichen Lithiumkonzentration von 11,5 $\mu \mathrm{g} / \mathrm{l}$ ausgesetzt waren (Interquartilbereich: 6,5 bis 14,9 $\mu \mathrm{g} / \mathrm{l})$. Signifikant höher lag der Wert bei Teilnehmern der Kontrollgruppe (12,2 $\mu \mathrm{g} / \mathrm{l}$; Interquartilbereich: 7,3 bis 16,0; $\mathrm{p}<$ 0,001). Allerdings war dieser Zusammenhang nicht linear. Denn im Vergleich zu Studienteilnehmern mit einer Lithiumexposition von 2,0 bis 5,0 $\mu \mathrm{g} / \mathrm{l}$ war das Inzidenzratenverhältnis (IRR) als Kenngröße bei jenen verringert, die regelmäßig mehr als 15,0 $\mu \mathrm{g} / 1$ (IRR: 0,83; < 0,001) und zwischen 10,1 und 15,0 $\mu \mathrm{g} / \mathrm{l}$ (IRR: 0,98; $\mathrm{p}=0$ 0,17) Lithium mit dem Trinkwasser aufnahmen. Erhöht war die Rate dagegen bei jenen mit Aufnahme einer Lithiummenge zwischen 5,1 und 10,0 $\mu$ g/l (IRR: 1,22; p $<0,001)$. Diesen Zusammenhang beobachteten die Wissenschaftler bei vaskulärer Demenz wie bei Demenz vom Alzheimer-Typ gleichermaßen. Allerdings können sie Einflüsse von ergeb- nisverzerrenden Faktoren nicht ausschließen.

Zwar herrscht unter den Wissenschaftlern im Zusammenhang mit dem biologischen Effekt von niedrigdosiertem Lithium keine Einigkeit. Doch wurde zumindest in Zellkulturen beobachtet, dass subtherapeutische Dosen des Leichtmetalls die Ausschüttung sowohl pro- als auch antientzündlicher Interleukine durch kortikale und hippocampale Neuronen, die in der Petrischale mit Gliazellen zusammengebracht werden, modulieren. Zudem beeinflussen geringste im Gegensatz zu höheren Dosen das Gleichgewicht der Zellmembranen. Unter anderem werden die Enzyme Phospholipase A2 im Zytosol sowie die kalziumabhängige Phospholipase 2 in Neuronen des Kortex und des Hippocampus aktiviert. Aus Tierversuchen ist bekannt, dass die Aktivierung dieser Enzyme für die Gedächtnisbildung und dessen Abruf erforderlich ist. (PL)

\section{Quelle}

Kessing LV et al. Association of Lithium in Drinking Water With the Incidence of Dementia. JAMA Psychiatry 2017, online 23. August. DOI: https://dx.doi.

org/10.1001/jamapsychiatry.2017.2362

\section{Nahrungsergänzungsmittel: Wann sind sie sinnvoll?}

\begin{abstract}
Nahrungsergänzungsmittel werden von vielen Menschen zur Vorsorge geschluckt. Ist das notwendig? Bei einer Pro-und-Kontra-Sitzung beim Kongress der Deutschen Gesellschaft für Innere Medizin (DGIM) konnten sich die Kontrahenten zumindest auf einen Minimalkonsens verständigen: Bei Vitamin D und Jod reicht die Ernährung in unseren Breiten zur Deckung des Bedarfs nicht aus, Schwangere brauchen natürlich Folsäure. Bei anderen Punkten herrschte Uneinigkeit.
\end{abstract}

» Nahrungsergänzungsmittel (NEM), die zu den Lebensmitteln zählen (genauer: ein Konzentrat von Nähr- oder sonstigen Stoffen mit ernährungsspezifischer bzw. physiologischer Wir- kung) sind nicht bei einem klinischen Mangel indiziert, betonte Prof. Hans Konrad Biesalski, Universität Hohenheim. Vielmehr dienen sie der Vorbeugung eines Mangels, in erster Linie bei
Menschen mit erhöhtem Bedarf an Mikronährstoffen oder eingeschränkter Zufuhr. Altbekannt, aber immer noch erwähnenswert, ist der erhöhte Bedarf von Schwangeren an Folsäure zur Verhinderung von Neuralrohrdefekten. Biesalski, der auf der Sitzung den ProPart übernahm, empfahl bereits bei Frauen mit Kinderwunsch die Folsäure-Supplementation. Er riet anstelle der standardmäßigen Folsäure-Supplementation zur Einnahme von Multivitaminpräparaten. In einer Studie wurde durch Gabe eines Multivitaminpräparats versus Placebo und Fol- 